\title{
Análisis cualitativo de la percepción de las pseudociencias en el colectivo médico español
}

\section{Qualitative analysis of pseudoscience's perception in Spanish healthcare professionals}

\author{
Bertran Salvador-Mata $a, b$, Valentina Raffio ${ }^{a, b}$, Sergi Cortiñas-Roviraa,b \\ a Departamento de Comunicación, Universitat Pompeu Fabra, España \\ b Grupo de Investigación en Comunicación Científica, Barcelona, España
}

\begin{abstract}
Resumen
Introducción: Las pseudociencias utilizan herramientas discursivas falaces para inmiscuirse en la sociedad. Estudios recientes investigan a diferentes colectivos profesionales para identificar, caracterizar y subvertir estos discursos. Objetivo: Analizar la percepción de las pseudociencias en el colectivo médico español. Método: Se usó una metodología cualitativa basada en entrevistas en profundidad. Los datos se analizaron mediante un modelo de análisis inductivo con la intención de identificar los patrones comunes presentes en las entrevistas. Resultados: Los entrevistados definieron las pseudociencias como manifestaciones carentes de método científico y que derivan de la autoridad de un supuesto especialista. Se muestran reticentes ante su uso. La connotación espiritual de las pseudociencias, junto con la instrumentalización del efecto placebo, su disfraz de natural e innocuo, y el acompañamiento emocional se identificaron como las causas principales de su expansión. Conclusiones: 1) para la prevención de las pseudociencias debe contarse con el apoyo coordinado de las autoridades sanitarias; 2) la precarización de los servicios asistenciales puede ser una de las causas de su expansión; 3) en el contexto de la posverdad, los argumentos emocionales 0 espirituales adquieren una mayor autoridad que los científicos, causando una pérdida de confianza en el marco epistemológico científico.
\end{abstract}

Palabras clave: pseudociencia; médicos; ciencia; dominios científicos; relación médico-paciente; percepción de la pseudociencia; sociología de la ciencia; comunicación social.

\begin{abstract}
Introduction: Pseudoscience's use fallacious arguments to expand in society. Different groups of professionals are being analyzed in order to identify, characterize and eventually undermine these major communicative patterns. Objective: To analyze the Spanish healthcare professionals' perception of pseudoscience. Methodology: A qualitative approach was used, consisting of in-depth interviews and questionnaires. Data was analyzed using an inductive analysis with the intention to identify the main patterns present in the subjects. Results: Interviewees defined pseudoscience as those practices lacking any scientific method and created by false specialists. The spiritual character of pseudoscience, together with the exploitation of the placebo effect, its disguise of natural and innocuous, and the emotional support of the preceptors of the false therapies are highlighted as the main causes of its spread. Conclusions: 1) For pseudoscience's prevention, healthcare professionals should be supported by coordinated strategies from the authorities; 2) the decline in public investment in the care services can be one of the causes of its expansion; 3 ) in the post-truth context, the emotional and spiritual arguments acquire a greater authority than the scientific ones, thus provoking a loss of confidence of the epistemological scientific framework.
\end{abstract}

Keywords: pseudoscience; healthcare professionals; science; scientific domain; physician-patient relation; pseudoscience perception; science sociology; social communication. 


\section{Introducción}

os avances científicos coexisten actualmente con la irrupción de su contrapunto negativo: la emergencia de las pseudociencias o falsas ciencias. La ciencia moderna, caracterizada por el empirismo y el racionalismo de Bacon y Descartes (Harman, 1971) aborda el problema epistemológico desde la definición de una metodología objetiva, experimental, que se desliga de cualquier misticismo o característica metafísica y niega el dogmatismo y la inmovilidad del saber (Bunge, 2013a). Para Bunge, el conocimiento científico debe cumplir una serie de características, entre las que destacan: la adaptación factual a la realidad ligada con la capacidad de ir más allá de los hechos y establecer teorías; el carácter analítico y especializado; el uso de su propio lenguaje compartido por la comunidad que le permite su comprobación, su verificación y, finalmente, su mejoría constante. Estas características propias del pensar científico fueron las responsables de grandes éxitos, como la conocida como la segunda revolución de la biomedicina (Ponti, 2001), y se espera que siga siéndolo, como se postuló en el editorial del Journal of American Medical Association (Nathan, Fontanarosa \& Wilson, 2001).

A la sombra del pensamiento científico emergen las pseudociencias, ganando mayor trascendencia en la sociedad de la posverdad, entendida como aquel contexto en que los argumentos emocionales son priorizados frente a los racionales, tal y como la define Oxford Dictionaries (2017). No siempre es sencillo establecer criterios de demarcación ni estructuras epistemológicas que logren discernirlos, y la sociología de la ciencia ha ido planteando distintos enfoques para definir y entender el proceso científico. Desde aproximaciones como las de Kuhn (1971), que plantean una relectura de la filosofía de la ciencia pasando de la ciencia inmadura a la consolidación paradigmática y a la posterior revolución contra ese paradigma, hasta el falsacionismo defendido por Popper (1989) o el anarquismo epistemológico. A pesar de la variedad argumental y filosófica, cabe enfatizar la necesidad de recuperar el sentido pragmático de la clasificación de la ciencia, en tanto que la expansión de las pseudociencias implica riesgos prácticos para la sociedad, aparte del empobrecimiento de la investigación y de la comunicación científica (Bunge, 2013b).

Alejándonos de los debates epistemológicos, en general se suele definir la pseudociencia como aquel conjunto de disciplinas que pretenden asemejarse a la ciencia sin utilizar el método científico a través de un proceso de mímesis parcial (Gordin, 2012). Como apunta Bunge (2013b), ciencia y pseudociencia son campos antagónicos: la ciencia se sitúa en la órbita del conocimiento y la investigación, constituyendo un corpus de conocimiento en constante progreso, mientras que la pseudociencia pertenece al campo de las creencias y se caracteriza por contar con teorías individuales para cada disciplina. A este respecto, las pseudociencias conforman un conocimiento estanco y hermético, que rechaza el resto del conjunto de saberes y no se adecua ni a la realidad factual ni a las teorías compartidas por una comunidad. Dichas teorías nacen de la autoridad de un experto usando el argumento ad autoritas, de modo que sus discursos recaen en la teoría y no en su demostración. El individuo adquiere autoridad singular en las pseudociencias a diferencia de la ciencia, cuyo corpus se fundamenta en la acumulación de conocimiento en cierto sentido anónimo.

Cuando las prácticas de falsa ciencia llegan al campo de la salud suponen un peligro. El colectivo médico debe enfrentarse a ellas a diario y por ello los profesionales sanitarios son fundamentales en la prevención de las pseudociencias. Estudiar los elementos discursivos de las pseudociencias resulta de capital importancia, en tanto que no se trata ya de un debate epistemológico sino de un problema de comunicación y utilización de argumentos falaces. El presente artículo corresponde a la continuación del proyecto de investigación de $1+D+i$ "Pseudociencia y sociedad en España. Análisis de las estrategias comunicativas usadas por la pseudociencia para su inserción social: métodos, discursos y usuarios" (CSO 2014-54614; 2015- 2017). Entre los colectivos analizados en este proyecto se encuentran farmacéuticos, prescriptores de pseudociencia, usuarios, universidades y periodistas científicos.

Este trabajo identifica específicamente el posicionamiento del colectivo médico frente a las pseudociencias, define posibles causas de su expansión, y esboza soluciones concretas desde el campo de la salud pública para hacerles frente.

\section{Métodos}

Esta investigación se estructuró en torno a dos preguntas de investigación: P1. ¿Qué percepción tienen los médicos acerca de las pseudociencias? y P2. ¿Qué situaciones identifican los entrevistados como causantes de la expansión de las pseudociencias? Se adoptó una metodología de investigación cualitativa, similar a la descrita por Cortiñas-Rovira, Alonso-Marcos, PontSorribes, Escribà-Sales (2014) y fundamentada en entrevistas en profundidad y cuestionarios. 


\section{Muestra experimental}

Se seleccionó una muestra de 31 profesionales de la medicina. El conjunto se caracterizó por su heterogeneidad para intentar conseguir representatividad. La selección es variada en cuanto a sexo, edad, años de experiencia, categoría profesional, grado de especialización y el tipo de contacto con los pacientes. La media de edad de los entrevistados se sitúa en 52,5 años, mientras que el promedio de experiencia es de 25,6 (datos completos disponibles en los archivos de la Universitat Pompeu Fabra).

\section{Entrevistas en profundidad}

Dividida en dos bloques, la entrevista consiste en preguntas semi-abiertas que permiten establecer una discusión estructurada. Las reuniones se han desarrollado en los despachos o las consultas de los profesionales, en su mayoría en Barcelona y su área metropolitana. La duración de las entrevistas se encuentra entre los treinta y los noventa minutos y han sido realizadas entre junio y septiembre de 2016. Con tal de complementar el resultado cualitativo, se han realizado dos encuestas enfocadas a contabilizar algunos de los conceptos clave del estudio. La primera se basa en la evaluación de unas afirmaciones relacionadas con la pseudociencia, mientras que la segunda está relacionada con la evaluación crítica de diferentes disciplinas pseudocientíficas. Ambas encuestas se han contestado siguiendo una escala de Likert y como complemento a la investigación cualitativa.

\section{Análisis de los datos}

Se estableció un modelo de análisis inductivo con la intención de identificar los patrones comunes detectados. Los más representativos se agruparon y se ordenaron para definir unos patrones discursivos y una argumentación común. La información de las encuestas se utilizó para perfilar los resultados cualitativos. La categorización y selección de los patrones se realizó: 1) de manera acordada entre los autores; 2 ) en función de la frecuencia de aparición de los mismos; y 3) en relevancia de los tópicos para responder a las preguntas de investigación. Esta investigación no pretendió obtener significancia estadística, sino definir los patrones argumentales comunes o compartidos por el colectivo médico, bajo la asunción que la heterogeneidad de la muestra permite representar los argumentos más comunes utilizados para definir o percibir las pseudociencias.

\section{Resultados}

\section{Definición de pseudociencia}

Para los entrevistados, el término pseudociencia engloba aquellas disciplinas que prometen unos resultados más allá de cualquier evidencia científica contrastada. Se detectaron dos ejes definitorios:
(1) La falta de rigor científico en la demostración de sus afirmaciones, que no derivan de ensayos clínicos representativos y realizados por métodos científicamente avalados: «Hay terapias que no están interesadas en pasar por un ensayo clínico. Si no están siguiendo el método para demostrar las cosas es que saben que en realidad no funcionan» (M9).

(2) La creación de una teoría no metodológica ni científica que las avala tanto médica como filosóficamente. Parte del colectivo afirma que las pseudociencias construyen un sistema ideológico a su alrededor en el que se transmiten valores como la paz interior, el equilibrio y la harmonía (M1), y que: "[utilizan] lo que llamamos documento de autoridad. Es decir, toda la terapia se basa en la opinión de un supuesto experto» (M18).

\section{Percepción de las pseudociencias}

El colectivo médico percibe el fenómeno de la pseudociencia con reticencia y preocupación. Una porción mayoritaria de la población analizada no consideró que pudiera definirse como estafa (Tabla 1, P1). Aun así, existen reticencias expresadas en cuanto a) no pueden afirmar ninguna evidencia contrastada (Tabla 1, P2); b) se fundamentan en el desconocimiento de la población y por tanto se aprovechan de él para sus argumentos (Tabla 1, P3) y c) se establece la desesperación como una de las herramientas que usa la pseudociencia para inmiscuirse en la sociedad (Tabla 1, P4).

La tabla 1 constata además que el fenómeno de las pseudociencias genera una fuerte polarización. El término medio (opción 3) es el menos utilizado, siendo la más votada siempre uno de los extremos (1/5).

\section{El concepto de terapias alternativas}

Para los profesionales entrevistados, las terapias alternativas representan un punto intermedio entre las disciplinas estrictamente científicas y las pseudociencias en tanto que intentan demostrar su eficacia, pero no pasan a través del filtro del método científico. La tabla 2 describe una jerarquía entre terapias donde se observa una gradación: la única manifestación científica (quimioterapia) adquiere un grado superior de rigor y efectividad, pero existe un conjunto de prácticas intermedias (acupuntura, osteopatía) que si bien no llegan al 4 sí superan el 1, convirtiéndose en terapias que el colectivo médico considera intermedias.

En relación a estas manifestaciones intermedias, la mayoría de los médicos sostuvieron que no existen estudios o ensayos clínicos de metodología empíricacientífica que confirmen la existencia de los conceptos utilizados por estas disciplinas para justificar su eficacia pero se les atribuye un cierto mérito debido al contacto entre terapeuta y paciente. 
Tabla 1. Resultados porcentuales de las preguntas hechas a miembros del colectivo médico en relación a su percepción para con las pseudociencias. Su numeración corresponde a la aparición en la tabla y no a su posición relativa en el cuestionario.

\begin{tabular}{|l|c|c|c|c|}
\hline P1: La pseudociencia supone un fraude económico $(n=31)$ \\
\hline $\begin{array}{l}\text { 1. Totalmente en } \\
\text { desacuerdo }\end{array}$ & 2. En desacuerdo & $\begin{array}{l}\text { 3. Ni de acuerdo ni } \\
\text { en desacuerdo }\end{array}$ & 4. De acuerdo & $\begin{array}{l}\text { 5. Totalmente de } \\
\text { acuerdo }\end{array}$ \\
\hline $51,61 \%$ & $12,90 \%$ & $9,67 \%$ & $9,67 \%$ & $16,12 \%$ \\
\hline
\end{tabular}

P2: Las terapias alternativas pueden ser consideradas como pseudociencia cuando prometen unos resultados más allá de cualquier evidencia contrastada $(n=31)$

\begin{tabular}{|c|c|c|c|c|}
\hline $\begin{array}{l}\text { 1. Totalmente en } \\
\text { desacuerdo }\end{array}$ & 2. En desacuerdo & $\begin{array}{l}\text { 3. Ni de acuerdo ni } \\
\text { en desacuerdo }\end{array}$ & 4. De acuerdo & $\begin{array}{l}\text { 5. Totalmente de } \\
\text { acuerdo }\end{array}$ \\
\hline $3,22 \%$ & $6,45 \%$ & $0 \%$ & $25,80 \%$ & $64,51 \%$ \\
\hline
\end{tabular}

\begin{tabular}{|c|c|c|c|c|}
\hline \multicolumn{3}{|l|}{ P3: La mayoría de pseudociencias se aprovechan del desconocimiento científico de la población $(\mathrm{n}=31)$} \\
\hline $\begin{array}{l}\text { 1. Totalmente en } \\
\text { desacuerdo }\end{array}$ & 2. En desacuerdo & $\begin{array}{l}\text { 3. Ni de acuerdo ni } \\
\text { en desacuerdo }\end{array}$ & $\begin{array}{l}\text { 4. De acuerdo } \\
\text { acuerdo }\end{array}$ \\
\hline $0 \%$ & $3,22 \%$ & $0 \%$ & $38,70 \%$ & $58,06 \%$ \\
\hline
\end{tabular}

\begin{tabular}{|l|c|c|c|c|}
\hline \multicolumn{4}{|l|}{ P4: La mayoría de pseudociencias se aprovechan de la desesperación de sus usuarios $(\mathrm{n}=31)$} \\
\hline $\begin{array}{l}\text { 1. Totalmente en } \\
\text { desacuerdo }\end{array}$ & 2. En desacuerdo & $\begin{array}{l}\text { 3. Ni de acuerdo ni } \\
\text { en desacuerdo }\end{array}$ & 4. De acuerdo & $\begin{array}{l}\text { 5. Totalmente de } \\
\text { acuerdo }\end{array}$ \\
\hline $0 \%$ & $3,22 \%$ & $3,22 \%$ & $38,70 \%$ & $54,83 \%$ \\
\hline
\end{tabular}

Tabla 2. Concepción de la validez de determinadas terapias alternativas por parte de exponentes del colectivo médico español $(n=31)$. La valoración se ha efectuado a través de una escala de Likert con las siguientes equivalencias: 1 nulo, 2 bajo, 3 aceptable, 4 alto, 5 óptimo.

\begin{tabular}{|l|c|c|}
\hline & Rigor científico & Efectividad \\
\hline Homeopatía & 1,87 & 1,87 \\
\hline Biomagnetismo & 1,36 & 1,43 \\
\hline Reiki & 1,73 & 2 \\
\hline Acupuntura & 2,64 & 3 \\
\hline Flores de Bach & 1,56 & 1,65 \\
\hline Quimioterapia (control) & 4,64 & 4,22 \\
\hline Feng Shui & 1,43 & 1,5 \\
\hline Tarot & 1,03 & 1,1 \\
\hline Astrología & 1,29 & 1,26 \\
\hline Osteopatía & 3,29 & 2,64 \\
\hline Psicoanálisis & 2,87 & 2,96 \\
\hline Cosmología & 1,12 & 1,12 \\
\hline
\end{tabular}




\section{Instrumentalización del efecto placebo}

Uno de los mayores puntos de consenso entre los profesionales entrevistados es la relación que se establece entre los supuestos beneficios de las pseudociencias y el efecto placebo. Los entrevistados identificaron una instrumentalización falaz del efecto placebo en las pseudociencias, que se aprovechan de esta circunstancia para justificar una efectividad que no han sido capaces de demostrar por estructuras epistemológicas causales. Se identificaron los siguientes pasos para la instrumentalización del efecto placebo: 1) creación y divulgación de una teoría que avala el tratamiento y satisface las preguntas del paciente; 2) dedicación de tiempo en las consultas para escuchar al paciente más allá de la historia clínica; 3) forjamiento de una relación entre paciente y terapeuta basada en la confianza y la autoridad del experto. Se estableció una estructura que permitirá instrumentalizar el efecto placebo, y generar la ilusión de efectividad.

\section{La pseudociencia como respuesta emocional}

La medicina, como encarnación del método científico, desvela y encuentra soluciones pero es incapaz de hallar todas las respuestas que el ser humano necesita. Los entrevistados consideraron que las pseudociencias encuentran su posicionamiento en el mercado en los huecosa los que aún no hallegado la medicina. El colectivo médico identificó como una de las principales razones de la existencia de la pseudociencia la necesidad humana de encontrar respuestas satisfactorias a problemas vitales: «(...) buscan soluciones a temas que no tienen solución. $\mathrm{O}$ buscan soluciones rápidas a problemas que requieren tiempo» (M17).

\section{La pseudociencia como "natural, innocua y espiritual"}

La gran mayoría de disciplinas pseudocientíficas se definen a sí mismas mediante términos relacionados con lo natural. Los entrevistados señalaron que esto provoca una falsa sensación de inocuidad que atrae a determinados ciudadanos hacia este tipo de alternativas pensando que, en el peor de los casos, no existe ningún riesgo asociado.

Algunos entrevistados señalaron que el papel actual de la pseudociencia está más cerca de una concepción espiritual de la existencia humana que de una disciplina científica dedicada a tratar las patologías que afectan a la salud del paciente: «Las manifestaciones pseudocientíficas están más cerca de la religión que de la ciencia» (M21).

Un $71 \%$ del colectivo entrevistado consideró que la pseudociencia asume características semejantes a las de la religión (Tabla 3).

Tabla 3. Resultados porcentuales de las preguntas hechas a miembros del colectivo médico.

P: La pseudociencia equivale a la religión de nuestra era, ya que proporciona refugio emocional para soportar los miedos y las inseguridades humanas $(n=31)$

\begin{tabular}{|c|c|c|c|c|}
\hline $\begin{array}{l}\text { 1. Totalmente en } \\
\text { desacuerdo }\end{array}$ & 2. En desacuerdo & $\begin{array}{l}\text { 3. Ni de acuerdo ni } \\
\text { en desacuerdo }\end{array}$ & 4. De acuerdo & $\begin{array}{l}\text { 5. Totalmente de } \\
\text { acuerdo }\end{array}$ \\
\hline $6,45 \%$ & $6,45 \%$ & $16,13 \%$ & $51,61 \%$ & $19,35 \%$ \\
\hline
\end{tabular}




\section{Penetración de las pseudociencias en la sociedad española}

Los encuestados coincidieron en que actualmente este fenómeno se encuentra en auge. La universalización del uso de internet fue considerado como una posibilidad para eliminar el filtro de los profesionales sanitarios, poniendo al abasto de los ciudadanos una infinidad de terapias no necesariamente avaladas por el método científico. Esta dinámica beneficia a las pseudociencias, ya que han aprendido a utilizar los mecanismos de la red para vender sus productos y llegar a un público cada vez más amplio.

Hoy en día un enfermo con conexión a internet se va directo para el mundo de las pseudociencias. No encuentran una página que explique lo que es profesional y lo que tiene un prestigio. Pone lo que es milagroso, lo que puede ir bien (M10).

\section{Riesgos de las terapias no avaladas científicamente}

La falta de estudios rigurosos de estas disciplinas impide afirmar la innocuidad de las prácticas pseudocientíficas. Se señaló desde el colectivo médico que el mayor riesgo de la pseudociencia es ofrecerse como una alternativa válida a las terapias demostradas científicamente. El abandono de un tratamiento científico puede conllevar a situaciones irreversibles. Ante esta situación se detectan cuatro escenarios posibles (1) la terapia alternativa resulta inocua, (2) la falta de estudios científicos sobre sus beneficios y efectos adversos hace que se desconozca si la terapia puede interferir con otros tratamientos y perjudicar la salud del paciente; 3) que el paciente puede discontinuar su tratamiento, con posibles consecuencias de gravedad, y 4) el mismo uso de la terapia alternativa comporta un riesgo para la salud del afectado.

Si te ofrecen un tratamiento que no ha sido debidamente estudiado, al final lo que está en juego es tu salud. Puede haber hasta riesgo vital. Si por ejemplo tienes cáncer y te niegas a seguir el tratamiento establecido, lo más probable es que acabes antes muerto. (...) Las pseudociencias no te garantizan nada (M18).

\section{Permisividad hacia la pseudociencia}

La actitud mayoritaria del colectivo médico ante un paciente que quiere utilizar pseudociencias es informarle de la falta de rigor científico de las mismas con tal de persuadirlos para utilizar terapias médicas. Ante estas situaciones, se detectaron tres tipos de actitud: (1) negar rotundamente el uso de cualquier terapia no avalada científicamente, (2) intentar pactar una vía de conciliación entre las terapias alternativas y el tratamiento científico convencional, 0 (3) ceder ante el paciente y permitirle usar en exclusividad terapias alternativas. Se mencionó la Ley de Autonomía del Paciente (establecida en la Ley 41/2002 del 14 de noviembre) según la cual no se pueden imponer las recomendaciones del médico por encima de la voluntad del paciente. Así, aunque exista una clara reticencia en la comunidad médica, no en todas las ocasiones puede conseguirse prevenir la pseudociencia. Los entrevistados destacaron como especialmente relevante en este sentido la patología que padezca el paciente.

Se identificaron conductas de resignación ante la posibilidad de permitir el uso de terapias no avaladas científicamente. Las únicas excepciones donde la opinión médica puede prevalecer son situaciones que 1) involucren menores, 2) los afectados no están mentalmente estables 0 3) cuando pueden resultar afectadas terceras personas.

Se reflexionó acerca de hasta qué punto esta permisividad basada en la Ley de Autonomía del Paciente es beneficiosa para el mismo paciente, ya que existen casos en los que el abandono de las terapias científicas provoca una situación irreversible.

\section{Discusión}

El presente estudio ha analizado cualitativamente el fenómeno de la pseudociencia a partir de las percepciones del colectivo médico. Si bien la muestra analizada puede ser objeto de futuras ampliaciones, esta investigación ha identificado una serie de aspectos que deben ser tenidos en cuenta para la prevención de las pseudociencias. El éxito de los estudios de esta índole recae en que permiten analizar elementos que suscitan interés 0 preocupación en los entrevistados para poder estudiarlos con detenimiento, hallando patrones discursivos y características transversales que atañen al fenómeno de la pseudociencia. A nuestro conocimiento, es el primer estudio que acomete la problemática desde un enfoque cualitativo centrado en el colectivo médico español.

Se ha observado que no existe un consenso absoluto entre los profesionales acerca de la definición, el riesgo y la envergadura del fenómeno de las pseudociencias. Como otros estudios han señalado (Cortiñas-Rovira, 2014; Alonso \& Cortiñas 2014), la amplitud terminológica y la tendencia a la polarización dificultan el estudio del fenómeno. Aunque se ha detectado una reticencia generalizada, el nivel de rechazo varía en función del clínico, de la patología del paciente, de cómo este se 
posicione y de las consecuencias que pueda tener para la práctica clínica. Existe consenso acerca del riesgo inherente de las terapias pseudocientíficas: no únicamente el propio riesgo per se que puedan tener, sino los riesgos secundarios que pueden comportar: abandono de terapias convencionales, malos seguimientos y adherencias a los tratamientos y pérdida de confianza en el médico.

\section{Falta de compromiso de los organismos especializados}

Aunque el médico realiza una labor de comunicación y persuasión para evitar en la medida de lo posible el uso de terapias pseudocientíficas, el paciente en ejercicio de su autonomía es libre de consumir los productos que considere oportunos. Parte del colectivo demanda que existan regulaciones que garanticen que las prácticas pseudocientíficas se anuncien de manera veraz y sin incurrir en falacias. La fácil difusión de estas prácticas hace que cuando los pacientes llegan al médico muchos dispongan de ideas prefiguradas, lo que dificulta la práctica clínica y puede llevar a actitudes de resignación 0 aceptación por parte del profesional sanitario.

Los médicos entrevistados plantearon la necesidad de reivindicar un liderazgo científico que guie a la población. Consideran que la sociedad está empezando a gestar un rechazo hacia los postulados científicos, como los de la OMS, las sociedades científicas o los colectivos médicos. Este rechazo ha llevado a deslocalizar la autoridad científica en terceros, que en ocasiones pueden ser profesionales de las pseudociencias. El descrédito otorgado a ciertas instituciones científicas proviene en mayor medida de la mala gestión de crisis sanitarias en el pasado, momento en el que se causó un exceso de alarmismo entre la población, como podría suponer el caso de la gripe A. Según algunos profesionales, esto ha derivado en una pérdida de autoridad.

Los autores del estudio concluyen que los organismos científicos deben, 1) contribuir al control de las prácticas pseudocientíficas de manera consensuada, como por ejemplo realizó el gobierno de Australia con la homeopatía (Commonwealth of Australia, 2017); y 2) establecer estrategias de alfabetización científica en la población para garantizar el espíritu crítico.

\section{Falta de tiempo asistencial}

Los entrevistados han identificado como una posible causa de expansión de las pseudociencias la falta de tiempo asistencial. Tras los recortes en sanidad, los profesionales sanitarios consideran que no disponen de tiempo suficiente para dedicar al paciente. En contraposición, las terapias pseudocientíficas han sabido utilizar este déficit para ofrecer una alternativa que supone un mayor acompañamiento al paciente. Esta característica, junto con la instrumentalización del efecto placebo y el acompañamiento emocional, ofrecen una solución ante la falta de tiempo asistencial. Se produce así un alejamiento del paciente del colectivo médico y la autoridad de este se va diluyendo, hasta el punto que las opiniones que el paciente identifica en otros canales de comunicación configuran un nuevo corpus de autoridad no científica.

Los autores concluyen que solucionar la masificación y la falta de tiempo asistencial puede repercutir en un mejor tratamiento y un retroceso de las pseudociencias.

\section{Contenido espiritual de la pseudociencia}

La pérdida de autoridad de la evidencia científica no se da únicamente en el contexto médico. La sociedad de la posverdad, entendida como aquella donde se priorizan argumentos emocionales frente a los racionales (Oxford Dictionaries, 2017), o la situación de una sociedad como la define Lyotard (2006) donde ya no caben los metarelatos, pueden ayudar a explicar que prácticas de falsa ciencia que ofrezcan un contenido espiritual y una teoría unificadora y sencilla de fenómenos complejos y múltiples se expandan con facilidad. La verdad, que en la ciencia moderna ostentaba características metodológicas, se ha diluido, facilitando que prácticas falaces lleguen a más gente. El colectivo médico analizado ha destacado el rol espiritual de la pseudociencia, que adquiere, como apunta Bunge (2013b), tintes de creencia. Si bien podría objetarse que no solo no es positivo, sino que es imposible eliminar la espiritualidad en el ser humano, lo que puede intentar combatirse a opinión de los autores es el uso fraudulento que se hace de ella, asociándolo a productos de aplicación médica que fracasan en su vertiente curativa.

\section{Conclusiones}

Se concluye que 1) para la correcta prevención de las pseudociencias, el profesional sanitario debe poder contar con el apoyo de autoridades y otros colectivos profesionales en estrategias coordinadas; 2) la precarización de los servicios asistenciales puede ser una de las causas de la expansión de las pseudociencias; y 3) en el contexto de la posverdad, los argumentos emocionales 0 espirituales adquieren una mayor autoridad que los científicos, generando así una pérdida de confianza en el marco epistemológico científico. 


\section{Financiación}

Este trabajo estuvo financiado por el Ministerio Español de Economía y Competitividad, bajo una beca de $\mathrm{R}+\mathrm{D}+\mathrm{i}$ (CSO 2014-54614; 2015 -2017).

\section{Referencias Bibliográficas}

Alonso, F., \& Cortiñas, S. (2014). La pseudociencia como (des) información tóxica. Una taxonomía para comprender el fenómeno y sus manifestaciones. Ámbitos: Revista Internacional de Comunicación, 24, 61-70.

Bunge, M. (2013a). La ciencia, su método y su filosofía. Buenos Aires, Argentina: Siglo XX.

Bunge, M. (2013b). Pseudociencia e ideología. Pamplona, España: Editorial Laetoli.

Commonwealth of Australia (2017). Review of Pharmacy Remuneration and Regulation. Retrieved from:http://www.health.gov.au/internet/ main/publishing.nsf/content/7E5846EB2D7BA299C A257F5C007C0E21/\$File/interim-report-final.pdf

Cortiñas-Rovira, S., Alonso-Marcos, F., Pont-Sorribes, C., \& Escribà-Sales, E. (2014). Science journalists' perceptions and attitudes to pseudoscience in Spain. Public Understanding of Science, 24, 450-465.

Gordin, M. D. (2012). The pseudoscience wars: Immanuel Velikovsky and the birth of the modern fringe. Chicago, London: The University of Chicago Press Books.

Harman, P. M. (1971). La revolución científica. Barcelona, España: Editorial Crítica.

Kuhn, T. S. (1971). La estructura de las revoluciones científicas. Madrid: Fondo de Cultura Económica.

Lyotard, J. F. (2006). La condición postmoderna. Madrid, España: Editorial Cátedra.

Nathan, D. G., Fontanarosa, P. B., \& Wilson, J. D. (2001). Opportunities for Medical Research in the 21st century. Journal of the American Medical Association, 285, 533-534.

Oxford Dictionaries (2017). Post-truth. In English Oxford Living Dictionaries. Oxford, UK: Oxford University Press. Retrieved from: https://en.oxforddictionaries. com/definition/post-truth

Ponti, F. (2001). Claude Bernard: primer of the second biomedical revolution. Nature Reviews Molecular Cell Biology, 2, 703-708.
Popper, K. R. (1989). Conjeturas y refutaciones: el desarrollo del conocimiento científico. Barcelona, España: Editorial Paidós. 